\title{
Frustração de Arestas em (3, 6)-Fullerenes
}

\author{
Diego S. Nicodemos ${ }^{1}$, Sulamita Klein ${ }^{2}$, Luerbio Faria ${ }^{3}$ \\ ${ }^{1}$ Colégio Pedro II / COPPE - Sistemas, Universidade Federal do Rio de Janeiro (UFRJ) \\ Rio de Janeiro - RJ - Brasil \\ ${ }^{2}$ COPPE-Sistemas, Universidade Federal do Rio de Janeiro (UFRJ) \\ Rio de Janeiro - RJ - Brasil \\ ${ }^{3}$ Departamento de Matemática - Universidade do Estado do Rio de Janeiro (Uerj) \\ Rio de Janeiro - RJ - Brasil \\ nicodemos@cos.ufrj.br, sula@cos.ufrj.br, luerbiodcos.ufrj.br
}

\begin{abstract}
Resumo. Grafos (3,6)-fullerenes são grafos planares, 3-conexos, cúbicos cujas faces têm tamanho 3 ou 6. Determinar o menor número de arestas a serem deletadas de um grafo de modo a obter um subgrafo gerador bipartido é conhecido na literatura [Došlić and Vukičević 2007] como o Problema de Frustração de Arestas. Neste trabalho, abordamos o Problemas da Frustração de Arestas em grafos (3,6)-fullerenes. Mostramos que todo grafo $(3,6)$-fullerene com $n$ vértices torna-se bipartido após a retirada de no máximo $\sqrt{\frac{4}{3} n}$ arestas.
\end{abstract}

\begin{abstract}
A (3,6)-fullerene graph is a cubic bridgeless plane graph with all faces of size 3 or 6 . Determining the smallest number of edges that have to be deleted from the graph to obtain a bipartite spanning subgraph is known in the literature [Došlić and Vukičević 2007] as the Bipartite Edge Frustration Problem. In this paper, we investigate the Bipartite Edge Frustration Problem in $(3,6)$-fullerene graphs. We show that every graph $(3,6)$-fullerene on $n$ vertices becomes bipartite after deleting at most $\sqrt{\frac{4}{3} n}$ edges.
\end{abstract}

\section{Introdução}

Segundo Došlić e Vukičević [Došlić and Vukičević 2007] uma aresta $e \in E$ é dita frustrada com respeito à bipartição $\left(V_{1}, V_{2}\right)$ de $V$ se ambas as extremidades de $e$ pertencem a mesma classe da bipartição. O menor número de arestas a serem deletadas de um grafo de modo a obter um subgrafo gerador bipartido é conhecido na literatura como o Problema da Frustração de Arestas e é representado pelo parâmetro $\tau_{\text {odd }}$.

Um grafo $(3,6)$-fullerene é um grafo planar, cúbico, 3-conexo cujas faces têm tamanho 3 ou 6. Pela Relação de Euler sabe-se que todo grafo $(3,6)$-fullerene possui exatamente 4 faces triangulares (faces de tamanho 3). O resultado central deste trabalho fornece uma cota superior para o Problema da Frustração de Arestas em grafos $(3,6)$ fullerenes e estende o resultado similar obtido para grafos fullerenes.

Teorema 1.1 Se G é um grafo (3,6)-fullerene com $n$ vértices, então $\tau_{\text {odd }}(G) \leq \sqrt{\frac{4}{3} n}$.

O restante do artigo está dividido da seguinte maneira. Na seção 2, discutimos conceitos específicos para a abordagem do Problema da Frustração de Arestas e introduzimos a ideia de remendos e fossos. Na seção 3, provamos o Teorema 1.1. 


\section{Preliminares}

As definições usadas neste trabalho são definições padrões. Para maiores detalhes o leitor é convidado a consultar [Bondy and Murty 2008].

O grafo dual de um $(3,6)$-fullerene é uma triangulação planar sem loops ou arestas múltiplas e todos os seus vértices têm graus 3 ou 6 . No dual de um $(3,6)$-fullerene os vértices de grau 3 são também chamados de vértices defeituosos.

Por toda esta seção, $G$ é uma triangulação planar com todos os vértices de grau menor que 6. Sejam um grafo $G$ e um conjunto de vértices $T \subseteq V(G)$ tal que $|T|$ é par. Uma $T$-junção de $G$ é um subconjunto $J \subseteq E(G)$ tal que $T$ é o conjunto dos vértices de grau ímpar em $G[J]$. Observe que se $T$ é o conjunto dos vértices de grau ímpar de $G$ e $J$ é uma $T$-junção de $G$ então $|T|$ é par (pois o número de vértices de grau ímpar em um grafo é sempre par) e cada vértice de $G-J$ tem grau par (pois retiramos de $G$ uma quantidade ímpar de arestas referentes aos vértices de grau ímpar e uma quantidade par de arestas referentes aos vértices de grau par). $O$ tamanho da menor $T$-junção de $G$ é denotado por $\tau(G, T)$.

Seja $\delta_{G}(X)$ o conjunto de arestas em um grafo $G$ com exatamente um vértice em $X \subseteq V(G)$. Um conjunto $C$ de arestas de $G$ é um corte de aresta de $G$ se $C=\delta_{G}(X)$, para algum $X \subseteq V(G)$. Sejam $X \subseteq V(G)$ e $T$ o conjunto dos vértices de grau ímpar de $G$. Um $T$-corte é um corte de aresta $\delta(X)$ tal que $|T \cap X|$ é ímpar.

Um empacotamento de $T$-cortes de $G$ é uma coleção disjunta $\delta(\mathcal{F})=\{\delta(X) \mid$ $X \in \mathcal{F}\}$ de $T$-cortes de $G$. Se $T$ é o conjunto dos vértices de grau ímpar de $G$, então denotaremos por $\nu(G, T)$ o tamanho do maior empacotamento de $T$-cortes de $G$. Uma inclusão sábia minimal é um conjunto dentre uma coleção de conjuntos que não contém qualquer outro conjunto da coleção. Dado um empacotamento de $T$-cortes, um $T$-corte $\delta_{G}(X)$ é uma inclusão sábia minimal quando $\delta_{G}(X)$ não contém qualquer $T$-corte do empacotamento de $T$-cortes. Uma família $\mathcal{F}$ é dita laminar se para cada par $X, Y \in \mathcal{F}$, tem-se $X \subseteq Y, Y \subseteq X$, ou $X \cap Y=\emptyset$.

Seja $G^{*}$ o dual de um $(3,6)$-fullerene. $O$ grafo $G^{*}$ não é bipartido, pois suas faces são todas triangulares. O grafo $G^{* \prime}$ obtido subdividindo as arestas de $G^{*}$ é bipartido, pois todas as suas faces têm tamanho 6 . Considere o grafo $G^{* \Delta}$ obtido, a partir de $G^{* \prime}$, adicionando três novas arestas dentro de cada face de $G^{* \prime}$, incidentes a cada um dos 3 vértices de grau 2. Chamamos $G^{* \Delta}$ de refinamento de $G^{*}$. Todos os vértices em $V\left(G^{* \Delta}\right)-$ $V\left(G^{*}\right)$ têm grau 6 em $G^{* \Delta}$, portanto se $D$ é o conjunto dos vértices defeituosos de $G^{*}$, então $D$ é também o conjunto dos vértices defeituosos de $G^{* \Delta}$.

O Lema 2.1 foi provado por Klein, Faria e Stehlik [Klein et al. 2012].

Lema 2.1 Para toda triangulação planar $G$ e todo subconjunto $T \subseteq V(G)$ tal que $|T|$ é par, $\tau(G, T)=\frac{1}{2} \nu\left(G^{\Delta}, T\right)$. Além disso, existe um empacotamento de T-cortes em $G^{\Delta}$ que é laminar, ótimo formado por inclusões sábias minimais.

Sejam $X \subset V(G)$ e $G[X]$ um subgrafo, 2-conexo, de $G$ tal que todas as faces de $G[X]$, exceto a face exterior, são triângulos. Um fosso de largura $k$ em $G$ ao redor do remendo $G[X]$ é um subconjunto de $E(G)$ definido como:

$$
\delta_{G}^{k}(X)=\bigcup_{i=0}^{k-1} \delta_{G}\left(N^{i}[X]\right) .
$$


Em particular, $\delta_{G}^{1}(X)=\delta_{G}(X)$. Se $\sum_{v \in X}(6-d(v))=d$, então $\delta_{G}^{k}(X)$ é um $d$-fosso de largura $k$, como mostra a Figura 1.
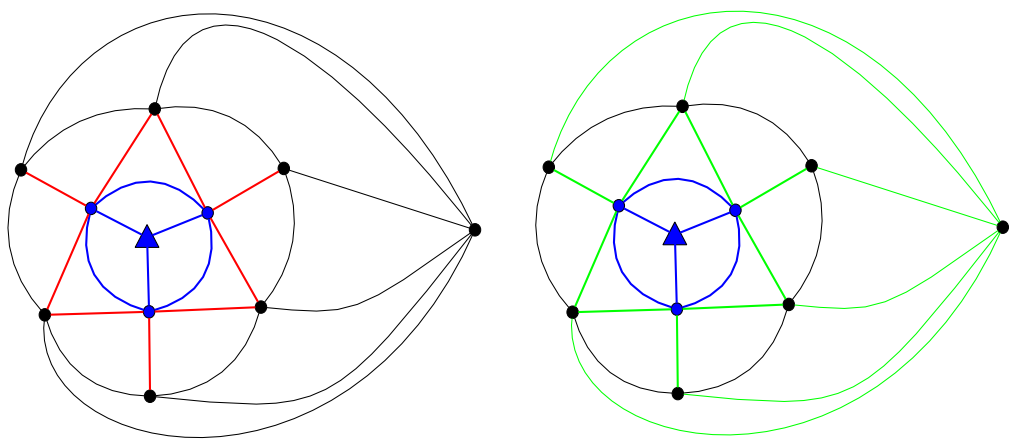

Figura 1. Nas duas figuras os subgrafos azuis representam remendos $G[X]$. As arestas de vermelho indicam um fosso de largura 1 e as arestas de verde um fosso de largura 2. Ambos são 3 -fossos.

Se $G^{*}$ é o dual de um $(3,6)$-fullerene, então os possíveis tipos de fossos de $G^{*}$ são os 3-fossos, os 6-fossos, os 9-fossos e os 12 -fossos.

Para todo fosso $\delta_{G}^{k}(X)$ corresponde um conjunto $\left|\delta_{G}^{k}(X)\right|$ de faces triangulares. Dizemos que as faces incidentes a pelo menos uma aresta de $\delta_{G}^{k}(X)$ são geradas pelo fosso $\delta_{G}^{k}(X)$. Se $G$ é um $(3,6)$-fullerene, então o número de arestas em um 3-fosso de $G^{*}$ é facilmente determinado.

Lema 2.2 Sejam $G$ um grafo $(3,6)$-fullerene, $G^{*}$ o dual de $G$ e D o conjunto dos vértices defeituosos de $G^{*}$. Se $d_{G^{*}}(u)=3$, e nenhuma aresta de $\delta^{k-1}(u)$ é incidente a vértices do conjunto $D-\{u\}$, então $\left|\delta_{G^{*}}^{k}(u)\right|=3 k^{2}$.

Demonstração. Observe que em cada camada do 3 -fosso de $G^{*}$ a quantidade de arestas é dada pela sequência $(3,3+6,3+6 \cdot 2,3+6 \cdot 3, \ldots, 3+6 \cdot(k-1))$. Portanto, $\left|\delta^{k}(u)\right|=\sum_{i=0}^{k-1}\left|\delta\left(N^{i}[u]\right)\right|=3 k+6(1+2+3+\ldots+k-1)=3 k^{2}$.

\section{Resultados Centrais}

O problema da frustração de arestas para os grafos $(3,6)$-fullerene será resolvido através de sua versão dual, ou seja, determinaremos o menor número de arestas a serem deletadas de um grafo $G$ de modo que o grafo remanescente não possua vértices de grau ímpar. Este parâmetro é denotado por $\tau(G)$. Já discutimos que quando $G$ é um grafo $(3,6)$-fullerene o seu correspondente dual $G^{*}$ é uma triangulação planar cujos vértices têm graus 3 ou 6 e existe pelo Lema 2.1, um empacotamento de $T$-cortes em $G^{* \Delta}$ que é laminar, ótimo, consistindo apenas de inclusões sábias minimais. Chamamos um tal empacotamento de um empacotamento de fossos de T-cortes que, neste caso, é composto apenas de 3 -fossos de $G^{*}$.

Teorema 3.1 Seja $G^{*}$ o grafo dual de um (3,6)-fullerene. Se $f$ é o número de faces de $G^{*} e T$ é o conjunto dos vértices de grau ímpar de $G^{*}$, então $\tau\left(G^{*}, T\right) \leq \sqrt{\frac{4 f}{3}}$.

Demonstração. Seja $G^{* \Delta}$ o refinamento de $G^{*}$. Assim $G^{* \Delta}$ é uma triangulação planar com $4 f$ faces e todos os vértices de graus 3 ou 6 . Pelo Lema 2.1, existe um empacotamento de fossos $\delta_{G^{* \Delta}}(\mathcal{F})$. Seja $m_{3}$ o número de arestas em um 3-moat de $\delta_{G^{* \Delta}}(\mathcal{F})$. Definimos o vetor de incidência $\vec{r} \in \mathbb{R}^{4}$ da seguinte maneira: para cada $u \in T$, seja $r_{u}$ a largura do 3-fosso centrado em $u$. 
Definimos o produto interno $\langle\cdot, \cdot\rangle$ em $\mathbb{R}^{4}$ por $\langle\vec{a}, \vec{b}\rangle=\sum_{u \in T} a_{u} b_{u}$. Também definimos a norma $\|\cdot\|$ por $\|\vec{a}\|=\langle\vec{a}, \vec{a}\rangle$.

Pela otimalidade de $\delta_{G^{* \Delta}}(\mathcal{F})$,

$$
\tau\left(G^{*}, T\right)=\frac{1}{2} \nu\left(G^{* \Delta}, T\right)=\frac{1}{2}\langle\vec{r}, \overrightarrow{1}\rangle, \text { sendo } \overrightarrow{1}=(1,1,1,1) .
$$

Suponha que $\delta_{G^{* \Delta}}^{r_{u}}(u)$ é um 3-fosso de $\delta_{G^{* \Delta}}(\mathcal{F})$, para algum $u \in P$. Lembre-se que pelo Lema 2.2, $\left|\delta_{G^{*}}^{r_{u}}(u)\right|=3 r_{u}^{2}$, logo somando sobre todos os 3 -fossos,

$$
m_{3}=3 \sum_{u \in T} r_{u}^{2}=3\|\vec{r}\|^{2}
$$

O grafo $G^{* \Delta}$ tem $4 f$ triângulos, e os 3 -fossos geram $m_{3}$ triângulos de $G^{* \Delta}$. Estes triângulos são mutuamente disjuntos.

Usando (2), temos: $4 f \geq m_{3} \geq 3\|\vec{r}\|^{2}$.

Consequentemente temos,

$$
\sqrt{\frac{4 f}{3}} \geq\|\vec{r}\|
$$

Portanto, por (1), (3) e pela desigualdade de Cauchy-Schwarz, segue que:

$\tau\left(G^{*}, T\right)=\frac{1}{2}\langle\vec{r}, 1\rangle \leq \frac{1}{2}\|\vec{r}\|\|1\|=\|\vec{r}\|$.

Concluímos que $\tau\left(G^{*}, T\right) \leq \sqrt{\frac{4 f}{3}}$.

Aplicando o Teorema 3.1 ao grafo dual, obtemos a prova do Teorema 1.1.

Prova do Teorema 1.1. Seja $G$ um grafo planar, cúbico, 3-conexo com $n$ vértices e com todas as faces de tamanho 3 ou 6 . O grafo dual de $G$ é uma triangulação planar com $n$ faces e todos os vértices de graus 3 ou 6 . Sejam $T$ o conjunto dos vértices de grau ímpar de $G^{*}, J^{*}$ uma $T$-junção mínima de $G^{*}$, e $J$ o conjunto de arestas de $G$ que corresponde à $J^{*}$. Como $G^{*}-J^{*}$ não tem vértices de grau ímpar, $G-J=\left(G^{*}-J^{*}\right)^{*}$ é bipartido, e pelo Teorema $3.1,|J|=\left|J^{*}\right| \leq \sqrt{\frac{4 n}{3}}$.

\section{References}

\section{Referências}

[Bondy and Murty 2008] Bondy, J. A. and Murty, U. S. R. (2008). Graph theory. Macmillan/Elsevier, Canada.

[Došlić and Vukičević 2007] Došlić, T. and Vukičević, D. (2007). Computing the bipartite edge frustration of fullerene graphs. Discrete Applied Mathematics, 155(10):12941301.

[Klein et al. 2012] Klein, S., Faria, L., and Stehlík, M. (2012). Odd cycle transversals and independent sets in fullerene graphs. SIAM Journal of Discrete Mathematic, 48(3):1458-469. 